\title{
L'IDENTITÉ DE L'OBJET : L'HÉRITAGE HUMIEN DE QUINE
}

\author{
LAYLA RAÏD
}

\begin{abstract}
RÉSUMÉ. Nous montrons comment Quine hérite de Hume certains aspects de sa conception de l'objet physique. L'un et l'autre, examinant l'étendue de la garantie empirique de nos concepts, soutiennent que l'identité de l'objet n'est pas suffisamment déterminée par l'expérience. Chez Quine, cette thèse est fondée sur l'indétermination de la référence, chez Hume sur une analyse de l'identité comme fiction de l'imagination. Quine, déterminant ainsi sa situation propre dans une histoire subjective de l'empirisme, voit cette analyse comme la perception confuse du fait que l'identité apparaît dans le système conceptuel seulement lors de l'acquisition de la référence divisée. Nous montrons comment les deux philosophes font alors face à une même tension, entre les exigences d'une construction empiriste des concepts à partir du matériau empirique et la dénégation d'une détermination complète. Nous montrons ainsi comment le rejet quinien du dogme du réductionnisme trouve une de ses racines dans le Traité de Hume.
\end{abstract}

\section{TABLE DES MATIÈRES}

1. Introduction : le développement de l'empirisme selon Quine $\ldots \ldots \ldots \ldots \ldots \ldots \ldots$. 1

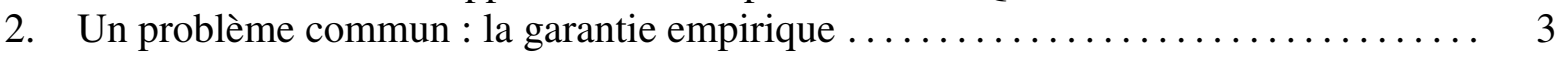

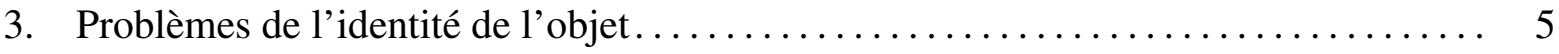

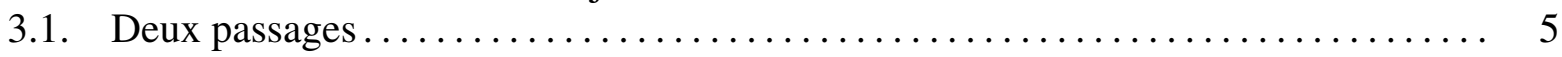

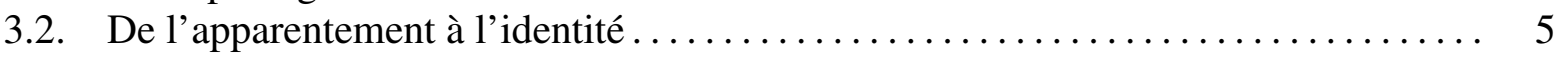

3.3. Termes de masse et termes à référence divisée $\ldots \ldots \ldots \ldots \ldots \ldots \ldots \ldots \ldots \ldots \ldots \ldots$

3.4. L'interprétation de Hume par Quine............................ 9

4. La naturalité de l'objet chez Quine et Hume : référence et coutume .......... 10

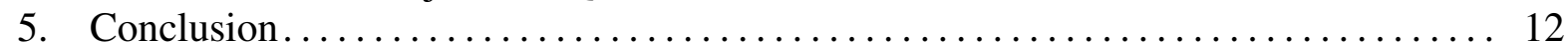

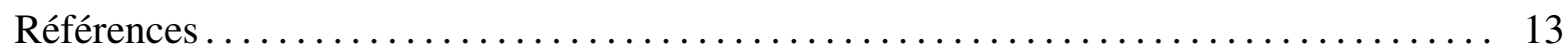

\section{INTRODUCTION : LE DÉVELOPPEMENT DE L'EMPIRISME SELON QUINE}

Dans «Five Milestones of Empiricism» [11], Quine propose une histoire à grands traits de l'empirisme et de ses amendements successifs, son système propre en constituant l'étape ultime. Les jalons de cette histoire sont présentés par un philosophe qui ne s'est jamais considéré comme un historien de la philosophie, mais qui s'est souvent réclamé d'une tradition empiriste ne remontant pas seulement à Russell et Carnap, mais bien à l'empirisme classique. Ces jalons caractérisent la manière dont Quine conçoit sa place dans l'histoire de l'empirisme, appelant ainsi à un travail d'appréciation des relations entre empirismes classiques et empirismes logiques. Ces five

\footnotetext{
* Version preprint. Paru dans la Revue de Métaphysique et de Morale 2003/2, p. 181-197.
} 
milestones présentent l'histoire de l'empirisme comme une progression, étape par étape, vers les positions holiste et naturaliste qui sont celles de Quine et correspondant à l'abandon de différents « dogmes » dénaturant les persuasions caractéristiques de l'empirisme; et, pour considérer le sens historique inverse, ils soulignent comment Quine lui-même situe ses travaux en continuité avec certains problèmes de l'empirisme classique.

La référence essentielle de Quine, même si on voit apparaître parfois les noms de Berkeley et Locke, est Hume; la parenté philosophique qui l'unit à ce dernier est souvent affirmée ; par exemple, elle est revendiquée, dans Roots of Reference :

Pour ce qui est de justifier l'induction, nous en revenons à Hume, aux côtés duquel nous nous rangeons sans aucun doute. [10, p. 20]

Dans le même texte, la critique de l'induction proposée par Hume, invoquée à titre d'argument définitif, est intégrée à ce que Quine nomme son naturalisme, défini comme le refus de toute philosophie première : la demande d'une justification de l'induction serait semblable à la demande d'une philosophie première qui viendrait au soutien de la science. Cette dimension immanentiste qui est une des caractéristiques du naturalisme de Quine, et qui relève d'une problématique différente de celle qui le mène par ailleurs au physicalisme ${ }^{1}$, est déjà présente chez Hume. Si l'appel à l'immanence est certes différemment construit chez l'un et l'autre, il est clair, dans de tels passages, que la philosophie de Hume a valeur inspiratrice pour Quine.

«L'épistémologie devenue naturelle », pour un second exemple, trouve un de ces points de départ non seulement dans l'Aufbau de Carnap, mais également dans le caractère définitif, selon Quine, des limites imposées à l'épistémologie du côté doctrinal, à savoir celles concernant la justification de nos connaissances sur la nature. «L'impasse humienne», écrit Quine, «est l'impasse humaine $»[8$, p. 86$]$; c'est du point de vue conceptuel qu'il y a eu progrès.

Ce progrès est donc résumé dans «Five Milestones of Empiricism» en quelques lignes. Notons que les étapes présentées dans «Fives Milestones» sont un développement de certains passages très proches de «Deux dogmes de l'empirisme» où Quine rappelle comment le réductionnisme était représenté par les philosophies de Hume et Locke, comment il était lié alors à une forme d'atomisme des idées, comment cet atomisme fut d'abord remis en question par la découverte des avantages ontologiques de la définition contextuelle chez Bentham ${ }^{2}$, puis chez Russell le nom de Frege apparaît aussi -, comment le réductionnisme devrait enfin être définitivement abandonné à l'issue des arguments proposés contre l'analyticité. Le même parcours historique apparaît également dans «L'épistémologie devenue naturelle».

\footnotetext{
${ }^{1}$ Cf. S. Laugier, L'anthropologie logique de Quine, sur la question de la nature et de l'articulation des différents éléments constituant le naturalisme de Quine, et en particulier sur l'évaluation des continuités et divergences entre naturalismes humiens et quiniens.

${ }^{2}$ Même si celle-ci est attribuée par Quine à Bentham, il cite son nom sans s'attarder spécifiquement sur la théorie des fictions; nous trouvons un renvoi indirect à l'ouvrage d'Ogden, Bentham's Theory of Fictions en note de « Five Milestones ». Second problème, la source directe de l'usage de la définition contextuelle par Russell est sans doute, explique Quine, plutôt venue des mathématiques; ce qui pose, pour cette histoire de l'empirisme, le problème des continuités réelles, question qui relève alors d'une enquête proprement historique. Ce récit d'amendements progressifs qu'aurait subi l'empirisme selon Quine a une fonction de positionnement historique, sans que le détail des influences réelles ait été exploré. Mais en tant que positionnement historique, ce récit est un objet à la fois pour l'historien et le philosophe, en ce qu'il manifeste et revendique l'existence d'une tradition.
} 
Parmi ces five milestones, le premier moment essentiel dans la réforme de l'empirisme est un «tournant linguistique $»$ où les idées sont remplacées par les mots, tournant qu'essentiellement n'a pas effectué Hume, et que Quine lie au nom de Tooke. Le deuxième moment déplace l'unité de sens du mot à la phrase, avec l'invention de la définition contextuelle. C'est, par-delà Bentham, essentiellement Russell qui a eu le mérite, d'après Quine, de la porter au rang d'outil philosophique fondamental, à travers la doctrine des symboles incomplets. Le troisième moment est le déplacement de l'unité de sens de la phrase au système des phrases; c'est le moment du holisme, lié étroitement chez Quine au rejet de la distinction entre analytique et synthétique, présenté comme le quatrième jalon. Le dernier moment, enfin, est celui du naturalisme, présenté comme issu de deux sources négatives : premièrement, le désespoir de définir l'ensemble des termes théoriques en termes phénoménaux, y compris par le biais de la définition contextuelle, désespoir qui est issu de la perspective holiste ; la deuxième source négative est, «un réalisme impénitent, le robuste état d'esprit du scientifique qui n'a jamais ressenti aucun scrupule au-delà des incertitudes négociables internes à la science $»[11$, p. 72].

Nous aimerions montrer, dans le cadre de cette tradition empiriste revendiquée par Quine, comment le problème de l'identité des objets physiques a conservé des caractéristiques communes de Hume à Quine, moyennant une reformulation précisément fondée sur des amendements linguistique et holiste.

\section{UN PROBLÈME COMMUN : LA GARANTIE EMPIRIQUE}

Montrons d'abord la communauté globale des questions avant d'examiner quelques textes où Quine se réfère au problème de l'identité de l'objet physique tel qu'il est posé par Hume.

Dire que les deux philosophes partagent un empirisme apparenté, c'est voir, avant tout, la similitude de leurs attitudes face aux difficultés que le point de vue empirique oppose à la constitution d'une notion donnée : il ne s'agit jamais de remettre en question le primat de la garantie empirique; si celle-ci entraîne un problème, les conceptualisations philosophiques ne peuvent le résoudre qu'en respectant ce primat.

À propos de l'identité des objets physiques, les deux philosophes posent donc le même problème fondamental : que pouvons-nous dire de cette identité au regard de la garantie empirique ? Ils concluent tous deux que l'identité n'est pas empiriquement garantie, Hume construisant le thème d'une erreur perceptive, et Quine celui d'une indétermination. Par-delà la différence des réponses, on peut souligner comment le passage de l'idée à la phrase et au système est en effet une étape décisive pour transformer l'absence de garantie chez Hume en une indétermination. L'absence de garantie entraîne l'indétermination selon Quine, dès lors que la relation à l'expérience n'est plus attribuée à l'idée, mais à la phrase prise dans un système, où des «ajustements compensatoires », selon l'expression de «Relativité de l'ontologie », sont alors pensables. L'indétermination de la référence ${ }^{3}$ suppose avant tout qu'on voie la différence de nature entre une phrase

\footnotetext{
${ }^{3}$ Cette thèse est située dans le cadre plus général de l'indétermination de la traduction, selon laquelle on peut traduire l'ensemble des phrases d'un langage étranger de plusieurs manières différentes, selon plusieurs manuels de traduction différents, de telle sorte qu'ils restent compatibles avec l'ensemble du comportement verbal, dans sa cohérence interne et dans son lien avec les stimulations non-verbales. La thèse la plus générale affirme donc l'indétermination de la traduction, qui s'étend à la signification et à la référence. La thèse particulière portant sur
} 
considérée comme un tout et une phrase considérée terme à terme. La relation à l'expérience se faisant par le biais de la phrase vue comme un tout inscrit dans un système de phrases, diverses interprétations deviennent possibles au niveau de la référence des termes eux-mêmes. Dans une note du chapitre 2 du Mot et la chose sur l'indétermination, Quine reconnaît à Russell d'avoir vu dans Signification et vérité que les object words sont en réalité des phrases occasionnelles, mais lui reproche d'avoir négligé le fait suivant : que «l'usage d'un mot comme phrase occasionnelle, pour déterminé qu'il soit, ne fixe pas l'extension de ce mot comme terme» [7, p. 92]. La conviction humienne du caractère empiriquement non garanti de l'identité est ici plus proche de la pensée de Quine que ne le sont, à cet égard, les persuasions russelliennes. Quine lui-même n' affirme pas trouver chez Hume une source à l'indétermination de la référence, mais les parentés des deux pensées méritent d'être soulignées : la thèse de Quine devient moins historiquement originale, quand on la relit dans la continuité du Traité de la nature humaine.

Dans un second ensemble de questions, devant cette dissociation de l'identité et de ce que l'expérience nous autorise à soutenir philosophiquement, Hume demande une explication de notre tendance naturelle à croire en cette identité. Quine propose un problème parallèle : une fois qu'on a montré que la garantie empirique ne suffit pas à fonder l'identité de l'objet, en laquelle, pourrait-il dire, nous croyons, il reste encore à expliquer comment nous parvenons à notre ontologie.

On peut remarquer à cet égard la proximité des structures du Traité de la nature humaine d'un côté et de l'autre du Mot et la chose : un double mouvement qui consiste à montrer l'absence de garantie empirique pour une notion d'un côté, et de l'autre à expliquer comment cette notion se construit de fait à partir de l'expérience, dans le langage chez Quine - c'est l'objet du troisième chapitre du Mot et la chose, «L'ontogénèse de la référence»-, et dans l'imagination chez Hume (cf. la structure de la section «Du scepticisme eu égard aux sens $»$ ).

Quine se demande comment nous parvenons à cet ensemble de croyances en l'objet physique de deux manières différentes. La première manière consiste à montrer un chemin possible pour la construction de l'identité, au sein d'une spéculation, selon le terme de Quine, sur l'acquisition des concepts fondamentaux par le jeune enfant. Cette spéculation ne se revendique pas comme une histoire réelle, mais comme une évaluation de la manière dont la garantie empirique pénètre le réseau des concepts. Le statut des spéculations psychologiques de «L'ontogénèse de la référence » est ainsi de montrer de quelle façon l'expérience détermine, ou ne détermine pas, nos concepts, en une œuvre qui est donc à proprement parler épistémologique, qui doit être lue comme une contribution au versant «conceptuel» de l'épistémologie. Quine cherche à présenter un certain cheminement possible de la garantie empirique à travers la pensée, question qui se distingue de celle d'une psychologie réelle de l'apprentissage des concepts. Nous ne considérerons pas ici le problème important du lien entre cette spéculation et la psychologie réelle, nous contentant de

la référence dit que la référence des termes d'un ensemble de phrases données d'un langage peut être reconstruite de plusieurs manières radicalement différentes, mais qui sont cependant compatibles avec les données empiriques (verbales et non-verbales). C'est l'exemple célèbre de «Gavagai », phrase qu'un indigène prononce au passage d'un lapin, et que le linguiste, s'il croit qu'elle porte sur quelque chose comme des lapins, est en réalité libre de traduire selon des ontologies toute différentes : «lapin», «stade de lapin», «partie non-détachée de lapin», etc., du moment que le lien à l'expérience, nécessairement global, est respecté. 
souligner la dimension épistémologique classique, pour ainsi dire, de «L'ontogénèse de la référence», ainsi que la manière dont nous devons par conséquent comprendre la naturalisation de l'épistémologie selon Quine : non pas un abandon de la question de l'évaluation du contenu empirique de la théorie pour la question de la constitution réelle des concepts, mais une utilisation de la seconde pour répondre à la première, quitte à avoir recours à la spéculation en absence d'un schéma assez général pour remplir ce but. Là-dessus, la continuité avec la méthode humienne est évidente.

La seconde manière dont on peut se demander comment on parvient à notre ensemble de croyances est de répondre au problème de la possibilité même d'avoir quelque chose comme un concept d'identité, étant donné le primat de principe accordé à la garantie empirique. Aussi bien Quine que Hume se demandent comment leur concept d'identité est possible dans le contexte qu'ils ont construit, Hume en posant le problème du caractère apparemment contradictoire de perceptions existant indépendamment de l'esprit, et Quine en posant le problème du «non-sens » de la référence quand elle est comprise du point de vue de la traduction. Il s'agit alors de résoudre l'énigme suivante : comment peut-on seulement se référer à quelque chose, si l'idée de référence est anéantie par la seule considération de la garantie empirique, et si nous restons pourtant fidèle au caractère ultime de la garantie empirique?

Considérons maintenant, après ces quelques parallèles, les textes de Quine relatifs au problème de l'identité de l'objet chez Hume. Nous examinerons dans un deuxième temps comment Quine entend constituer la référence en concept bona fide.

\section{Problèmes de L'IDEnTité de L’obJeT}

3.1. Deux passages. A propos de la question de l'identité de l'objet, il existe au moins deux passages différents où Quine propose une référence explicite à Hume, à la fois pour affirmer une filiation, et pour montrer quelles confusions, selon ses termes, on doit reconnaître chez lui. Nous considérerons d'abord «Identity, Ostension and Hypostasis», publié dans From a Logical Point of View; ensuite le paragraphe 24 du Mot et la chose intitulé «L'identité», situé dans le chapitre sur l'ontogénèse de la référence.

3.2. De l'apparentement à l'identité. Dans «Identity, Ostension and Hypostasis », Quine indique une différence essentielle entre deux manières de faire référence à un phénomène donné, en prenant, non sans ironie, l'exemple de la rivière d'Héraclite, où on ne se baigne jamais deux fois. Il rappelle ainsi le jeu sur les conditions d'identité que constitue cette sentence. On peut premièrement faire référence à des objets momentanés : à deux stades momentanés de la rivière, distingués par un certain intervalle de temps. Leurs relations sera celle d'une simple parenté, selon le terme ici utilisé par Quine de kinship. Dans ce premier cas, la rivière en tant qu'objet doué d'une identité propre n'est pas encore constituée. Nous disposons simplement d'un ensemble d'objets momentanés simplement apparentés les uns aux autres : nous disposons d'une river kinship. On peut deuxièmement faire référence à la rivière par-delà tous ces divers objets apparentés entre eux, en la posant comme un objet perdurable, c'est-à-dire étendu dans le temps, doué d'une identité à part entière. Il faut ainsi distinguer deux concepts : la « river kinship » et la «river» tout 
court. Quine souligne alors que ces remarques ont une parenté évidente avec l'analyse humienne, dont il rappelle quelques éléments.

Selon le Traité sur la nature humaine ( «Du scepticisme eu égard aux sens »), l'identité de l'objet physique est une fiction construite pour pallier l'interruption toujours renouvelée de nos perceptions. À partir de l'expérience de perceptions diverses, nous glissons insensiblement à l'idée de leur identité, quand elles se ressemblent assez, alors que l'interruption entre deux ensembles de perceptions nous engage au contraire à croire qu'ils sont distincts. La perplexité qui naît de cette contradiction, explique Hume, nous engage à unifier ces apparences brisées en posant la fiction d'une existence continue de l'objet, par-delà l'interruption manifeste de la perception. L'identité étant définie comme le caractère invariable et ininterrompu de l'objet, à travers une variation supposée du temps, selon les mots de Hume, on pose de manière erronée une identité de l'objet, malgré la simple ressemblance des perceptions entre elles, et malgré les interruptions de la perception. Quine rappelle ainsi comment l'identité de l'objet physique vient à être considérée par Hume comme une erreur au sens propre. Mais la communauté de point de vue qu'il juge importante porte sur la différence entre perceptions de ressemblances et position d'objets durables, les premières relevant d'un domaine de description précédant la maîtrise de l'identité de l'objet, et les secondes étant caractéristiques au contraire de cette maîtrise :

L'accusation par Hume d'une identification erronée est ici intéressante à titre de conjecture psychologique sur les origines, mais nous n'avons pas besoin de partager cette conjecture. Le point important à observer est simplement la connexion directe entre l'identité et la position de processus, ou d'objets étendus dans le temps. [6, pp. 66-67]

Ces remarques de Quine appelleront deux directions de développement différentes dans la suite de son œuvre. Deux ensembles de questions se croisent en effet ici : celle de la position d'identité à partir de ressemblances d'un côté, et de l'autre celle de la position d'un objet comme quelque chose de durable. La seconde question distingue entre le type de choses auxquels on fait référence : des rivières d'un côté, des ensembles apparentés d'objets distincts et momentanés de l'autre. Le type des objets reliés par la river kinship est essentiellement différent de ces «processus », qui deviendront plus tard, dans le Mot et la chose, la catégorie fondamentale où penser l'objet physique dans le langage enrégimenté (cf. § 36 sur le temps et le «parcage» (confinement) des termes généraux, où est repris le problème de la nature de l'objet «rivière » à partir de la sentence d'Héraclite). La première question porte sur la différence entre un réseau de ressemblances et un objet à part entière : c'est le propos du chapitre 2 «Traduction et signification » du Mot et la chose que de souligner cette différence, à partir de la construction d'ensembles de stimulations (le pendant quinien des ensembles de perceptions de Hume), dont on montre qu'ils ne suffisent pas à garantir la référence ${ }^{4}$. C'est cette différence qui attirera notre attention chez Quine, bien que la comparaison entre objets momentanés et perdurables, et en général la relation entre identité et temporalité, soit une dimension importante de la relation de filiation entre les philosophies de Hume et Quine.

\footnotetext{
${ }^{4}$ Nous nous intéressons ici à la seule question de l'identité de l'objet, et pas à celle de l'identité de signification, qui n'est pas un problème humien. Mais la position même du problème de l'indétermination de la signification a une facture humienne : sont distingués d'un côté des ensembles de stimulations et de l'autre des ensembles de pseudo-entités, en l'occurrence, qui ne peuvent trouver une identité à partir des stimulations.
} 
Chez Hume, la différence entre perceptions ressemblantes et perceptions d'objet aboutit au renvoi de l'identité dans le domaine des fictions de l'imagination. Quine, quant à lui, nous montre pour l'instant, dans «Identity, Ostension and Hypostasis », seulement la différence entre postuler une identité pour donner un nom à un ensemble d'objets semblables, par-delà cette seule relation de ressemblance, et postuler les objets formant cet ensemble. Cela n'implique aucun doute quant à cette identité elle-même; celle-ci est simplement distincte de l'identité d'un ensemble d'objets apparentés. On peut décider, en effet, de prendre un groupe raisonnable de ces ensembles comme la définition de notre objet. Ici, la position d'une identité apparaît comme le simple ajout d'une entité de nature différente, à partir d'objets déjà donnés. L'intérêt de cet ajout, affirme Quine, peut être la simplicité, et ainsi la plus grande maniabilité des concepts.

Dans la perspective du Mot et la chose, la distinction entre la river kinship et la river pourra être relue à la lumière d'une autre distinction que nous venons d'évoquer, et qui va radicaliser la position de Quine : celle entre un usage global du langage conditionné par des ensembles de stimulations et un usage analysé du langage où l'on passe du conditionnement à la référence. Ainsi l'apparentement des «objets momentanés » de «Identity, Ostension and Hypostasis » pourra être relu sous un autre angle : celui de la problématique de l'appariement entre ensembles de stimulations et de comportements verbaux. On trouve déjà dans cet article d'ailleurs l'idée que l'ostension de la rivière laisse ouvert d'abord un éventail d'interprétations possibles de ce qui comptera comme une « rivière », problème qui sera résolu ensuite inductivement, la rivière étant l'induction la plus raisonnable à propos de l'emploi de ce mot. La problématique de l'indétermination de la référence, posée plus tard ${ }^{5}$, parviendra précisément au rejet de l'idée qu'une procédure inductive pourrait fixer la référence.

3.3. Termes de masse et termes à référence divisée. Considérons maintenant Le mot et la chose, où Quine analyse les explications par Hume de l'identité. Nous y trouvons deux ordres de remarques. Elles contiennent, selon Quine, une confusion du signe et de l'objet, que nous examinerons dans un deuxième temps, pour nous concentrer d'abord sur la première remarque. Les explications de Hume expriment, d'après cette remarque, la perception d'un point fondamental de l'acquisition du concept d'identité selon Quine : à savoir que l'identité apparaît seulement quand le schème des objets est saisi.

Dans le troisième chapitre du Mot et la chose, «L'ontogénèse de la référence », Quine s'intéresse donc aux conditions d'acquisition de l'identité. Il existe une différence essentielle à cet égard dans l'apprentissage du langage : celle entre le conditionnement à réagir de la même manière à un ensemble de stimulations, d'un côté, et de l'autre la capacité à référer à des objets. L'identité n'apparaît que lorsque la deuxième capacité est acquise. Ainsi peut-on voir de deux manières différentes la reconnaissance d'une même chose par un enfant selon le stade où il se trouve dans l'apprentissage de l'appareil référentiel de son langage : un énoncé comme «Une rivière » peut signifier une réaction de l'enfant, similaire devant un ensemble de stimulations

\footnotetext{
${ }^{5}$ Nous renvoyons ici au Mot et la chose, même si l'expression même d'indétermination de la référence appartient à La poursuite de la vérité, où Quine amendera le vocabulaire du Mot et la chose. La référence y était dite inscrutable d'un point de vue empirique, mais pas indéterminée. Le point plus général de Quine dans La poursuite de la vérité est qu'aucune des deux notions, signification et référence, ne peut être empiriquement déterminée. Mais, pour notre propos, nous pouvons considérer que l'indétermination de la référence est une thèse du Mot et la chose.
} 
similaires. Pour prendre les termes de Hume, nous en sommes ici simplement au stade des perceptions ressemblantes. Une deuxième manière d'exprimer la reconnaissance de la rivière est l'usage d'un terme singulier, dans le cadre d'une maîtrise de l'appareil référentiel. Alors la prononciation de «Une rivière $n^{n}$ 'est plus directement reliée à la présence de certaines stimulations ; nous passons du conditionnement à la maîtrise de l'identité. C'est ici seulement que le mot «une » de l'expression prend tout son sens.

Parmi les outils utilisés pour expliquer cette différence, Quine propose un usage curieux de la distinction linguistique classique entre termes de masse et termes à référence divisée. Rappelons que les premiers ont la propriété de référer cumulativement : «toute somme de parties qui sont de l'eau est de l'eau» (cf. les usages des expressions «du sucre», «le mobilier», etc.). Ce n'est pas le cas des termes à référence divisée (les parties des choses auxquelles on réfère ainsi ne sont plus des références possibles pour ces mêmes termes) : soit le terme «pomme» dans «une pomme», «la même pomme», «une autre pomme», etc. Quine donne l'exemple de la célèbre comptine, ambiguë à cet égard : «Mary had a little lamb», qui signifie «Mary avait un petit agneau », si l'expression est un terme à référence divisée, et «Mary prit un peu d'agneau », si c'est un terme de masse. En quoi cette distinction intéresse-t-elle notre problématique ? C'est que Quine considère que les termes de masse sont une bonne approximation de la manière dont les termes du très jeune enfant se rapportent aux événements de sa vie. Ces «termes», comme «rouge », « eau », etc., sont en réalité utilisés, à l'état naissant du langage, comme des phrases ; mais si on veut les rapprocher du fonctionnement de véritables termes, alors :

(...) c'est peut-être à la catégorie des termes de masse qu'il est le plus tentant de rattacher ces termes en gestation, précisément parce que cette catégorie est flottante par rapport à la dichotomie sophistiquée du singulier et du général. [7, p. 143]

Ce sont ainsi les termes dont l'usage est le plus proche du simple conditionnement à produire un son devant une stimulation répétée. La véritable maîtrise de la référence naît à partir du moment où on divise la référence, de telle sorte qu'on est amené à distinguer des expressions comme «la même pomme», «une autre pomme», «différentes pommes», etc. Les termes de masse, quant à eux, ne demandent pas, dans leur emploi de termes de masse, une telle division : les expressions «de l'eau», «plus d'eau», «moins d'eau» peuvent être adéquatement employées pour toutes les stimulations aqueuses, sans que se pose le problème de la différence entre un objet et un autre, que nommerait le même terme. Le terme de masse n'est ainsi pas à proprement parler, pour Quine, un terme général, parce qu'il ne participe qu'imparfaitement à la grammaire des termes généraux. Et la maîtrise de l'identité est concomitante à celle de l'emploi de ces derniers. Quine nous propose donc une illustration de la différence entre conditionnement et discours référentiel à partir de la différence linguistique entre termes de masse et référence divisée. C'est là un usage proprement philosophique pour cette distinction qui par ailleurs est l'objet des linguistes. La référence cumulée est ainsi pour Quine une survivance de la période de conditionnement, un archaïsme linguistique. À l'objection naturelle selon laquelle les termes de masse réfèrent bel et bien, puisqu'ils réfèrent cumulativement, Quine répondra, dans le paragraphe sur la prédication (§ 20), que le comportement instable des termes de masse, parfois prédicat ( $«$ Le reste de la cargaison était du mobilier»), parfois sujet («L'eau coule»), nous conduit à abandonner la catégorie 
en question pour celle de terme général (premier exemple), ou celle de terme singulier (deuxième exemple).

3.4. L'interprétation de Hume par Quine. Considérons maintenant l'interprétation proposée par Quine de la construction humienne de l'identité comme motivée au fond par une perception confuse de l'idée suivante : il faut la référence divisée pour avoir un réel concept d'identité. Citons Le mot et la chose:

[Des] difficultés concernant l'identité forment l'arrière-plan de cette affirmation de Hume : «Nous ne pouvons dire, en aucune acception correcte des termes, qu'un objet est le même que lui-même, à moins que nous ne voulions dire par là que l'objet existant à un certain moment est le même que cet objet existant à un autre moment ». Il est probable que cette affirmation ait été inspirée à Hume en partie par ce que nous avons remarqué trois alinéas plus haut, à savoir par le fait que les énoncés d'identité reliant de simples termes fonctionnent à vide tant que le schème des objets physiques n'a pas été saisi. Mais il y a une autre cause, décelable en toute clarté dans les pages de Hume : si l'on comprend l'identité strictement comme la relation que chaque objet a exclusivement avec lui-même, on sera bien en peine de voir ce qu'elle a en elle de relationnel et en quoi elle diffère de la simple attribution d'existence. Or à la racine de cette difficulté, il y a une confusion entre le signe et son objet. Si l'identité est bien une relation, et $\ll=»$ un terme relationnel, ce n'est pas parce que l'identité relie des objets distincts mais parce que le signe $«=»$ se trouve entre des occurrences distinctes de termes singuliers, qui sont tantôt les mêmes termes, tantôt des termes différents. [7, p. 173]

Comment comprendre ces propos de Quine ? D'abord on peut insister sur une claire analogie déjà relevée entre les deux démarches. Le primat de la ressemblance sur l'identité chez Hume rejoint tout à fait la persuasion de Quine : pour ce dernier, le concept premier en philosophie est celui de similarité perceptuelle; Roots of Reference par exemple commence son premier chapitre avec les similarités perceptuelles pour parvenir ensuite à la position de l'identité, dans le chapitre suivant «Breaking into language $»$.

La remarque de Hume que cite Quine appartient au passage du Traité de la nature humaine où il propose une définition de l'identité. Rappelons-en quelques éléments. Il s'agit pour lui de comprendre comment on peut seulement parler d'identité, concept qui lui apparaît comme paradoxal. Un objet singulier exprime l'unité et pas l'identité, puisque dire «Un objet est le même objet que lui-même», c'est ne rien dire, car, selon les mots de Hume, «objet» et «lui-même» signifient la même chose. Par ailleurs, une multiplicité d'objet n'exprimera pas non plus bien entendu l'identité. Nous avons donc besoin pour penser l'identité de trouver une différence là où n'en voyions pas, une différence dans l'objet singulier. Celle-ci est donnée selon Hume par la perception de la succession temporelle. On perçoit le temps grâce à la succession de perceptions différentes ; mais on a coutume aussi de percevoir un objet qui ne subit aucun changement comme s'il était dans le temps, ce qui est une première fiction. On peut comprendre à partir de là l'identité comme celle qui existe entre un objet à un moment et à un autre de sa perception étendue dans le temps. L'identité est ainsi comprise comme le caractère invariable et ininterrompu de l'objet. L'identité attribuée à l'objet physique est encore une nouvelle fiction. 
Maintenant, cette conception de l'identité comme réclamant une succession temporelle pour pouvoir être dite a sans doute son origine, c'est la lecture peu orthodoxe de Quine, dans une perception confuse de la nécessité d'une référence divisée pour pouvoir vraiment parler d'identité. Quine accorde qu'il faut des différences pour qu'on puisse parler d'identité, mais la différence qui importe n'est pas temporelle. Quine renvoie ici au problème général du type de relation qu'est l'identité, relation qui doit être à la fois telle qu'on puisse montrer le caractère informatif d'un énoncé d'identité, et telle cependant qu'il s'agisse toujours bel et bien du même objet. Cette différence, que Hume tente d'installer dans le temps, est donnée selon Quine par la référence divisée, qui existe dans le langage. Quine examine au $§ 24$ du Mot et la chose l'idée que l'identité puisse être vraie de deux termes qui seraient simplement conditionnés par le même ensemble de stimulations frappant la même personne. Mais dans ce cas, par ailleurs improbable, l'énoncé d'identité ne fournirait précisément aucune information nouvelle. En réalité, on se trouverait dans la situation présentée par Hume (mis à part le fait que Hume ne distingue pas entre les termes et l'objet), où nous ne parviendrions pas à dire ce qui différencie les accès à l'objet, puisque précisément nous avons un et unique ensemble de perceptions. Chez Quine, si l'identité doit être informative, elle ne peut être expliquée à partir des seules stimulations (ni donc de la seule catégorie des termes de masse). Il trouve ainsi chez Hume, pour qui la simple perception d'un objet n'est pas encore position d'identité, une anticipation de cette idée.

Le deuxième point que Quine reproche à Hume tient à sa confusion du signe et de l'objet. On peut attribuer cette confusion au fait que Hume raisonne sur des perceptions, dont il considère la distinction d'avec les objets comme illusoire d'un point de vue empirique. Si c'est la perception qui est porteuse de référence, si on peut dire, alors on ne voit pas effectivement comment on pourrait éviter la confusion entre le signe et l'objet. Dans ce cas, l'identité doit être comprise comme Hume le fait, c'est-à-dire en cherchant l'origine de la différence au sein même des perceptions, par exemple au sein d'une perception inchangée se prolongeant dans le temps. Ici, la différence fondamentale de Hume à Quine est bien le premier pas du tournant linguistique, à savoir le passage des idées aux termes.

\section{LA NATURAlité DE L'obJet CHEZ Quine ET Hume : RÉFÉRENCE ET COUTUME}

Ces considérations sceptiques sur l'identité de l'objet physique conduisent, comme nous le rappelions plus haut, à des problèmes de cohérence chez les deux philosophes. Il s'agissait de constituer malgré tout l'identité au sein d'un système qui semble au premier abord ne pas pouvoir l'accueillir. Chez Quine, le problème est celui de la possibilité même de la référence, par-delà son indétermination.

Il semble que cette source de la philosophie de Quine qu'est Hume peut nous aider à comprendre le sens de certaines déclarations problématiques de «Relativité de l'ontologie» dont le rôle est de mettre fin à la ronde des doutes sceptiques à l'égard de la référence. Voici le paradoxe exposé par Quine dans «Relativité de l'ontologie», une fois présenté l'effondrement de la référence dans la traduction radicale :

«Nous voici apparemment en train de nous enfermer nous-mêmes dans la doctrine absurde qu'il n'y a pas de différence, quelle qu'en soit la nature, interlinguistique ou intralinguistique, objective ou subjective, entre référer à des lapins et référer à des parties de lapin (...). Nul doute 
que ce soit absurde, car cela entrainerait qu'il n'y a aucune différence entre le lapin et chacune de ses parties (...) Apparemment la référence deviendrait non-sens, non pas seulement dans la traduction radicale, mais chez nous (at home).» [9, pp. 60-61]

Quine fait référence à la fin de ce passage au fait que toutes les conséquences de la traduction radicale sont valables at home, c'est-à-dire à l'intérieur d'une même langue : il suffit de voir la compréhension de son voisin comme une traduction de ses phrases... Le problème est donc : quelle description donner de la manière dont nous référons tout de même à des objets? Il semblerait que le primat de la garantie empirique posé par Quine l'enferme dans un dilemme : la référence étant indéterminée au regard de la garantie empirique, elle ne peut, voudrait-on objecter, que perdre son sens.

Quine répond en proposant une notion « relative» (selon son expression) de la référence : la référence est effectivement une notion absurde, si on ne la rapporte pas à un langage d'arrière-plan. Si on demande par exemple «Que sont les objets physiques?», la réponse, pour être douée de sens, utilisera des termes dont la référence elle-même ne sera pas questionnée. Si on poursuit par contre indéfiniment la question, il est clair que la question elle-même finira par perdre tout sens. Le langage d'arrière-plan doit être accepté sans question ultérieure. À la relativité correspond l'idée, du point de vue psychologique, d'un acquiescement au langage, d'un assentiment, pour reprendre un terme humien, qui consiste à le comprendre au pied de la lettre. Ainsi la question de ce qui existe ne peut être posée véritablement du point de vue de la traduction radicale. Si dans la traduction radicale donc, on est amené à dire qu'il n'y a pas de différence entre référer à des lapins et à des parties de lapin, nous ne pouvons pas pour autant conclure qu'il n'y a pas de différence entre les références tout court, car nous ne pouvons déterminer des existences que depuis notre langue prise at face value. Le point est le suivant : nous ne pouvons pratiquer le jeu de la réalité qu'au sein d'un langage accepté comme tel, et ainsi l'absence de garantie empirique n'implique aucun renoncement à la référentialité.

Le problème que peut se poser le lecteur de Quine est alors celui du statut, déconcertant, de cet acquiescement à notre langage naturel. Est-ce autre chose qu'une rustine conceptuelle destinée à sauver un système appelant en réalité à un modus tollens, qui réfuterait en général l'empirisme de Quine?

Nous pouvons rapprocher, à cet égard, ces réponses de Quine de la distinction humienne entre philosophie et croyance coutumière. On voit en quoi la réponse de Quine a des affinités importantes avec celle de Hume (et d'ailleurs le même caractère déconcertant...) quand il s'agit de savoir ce que nous allons faire une fois détruite l'apparence de fondation de nos objets de croyance. La communauté d'esprit réside dans l'idée que toute forme d'affirmation de réalités se fera sur un mode naturel de croyance, opposé au mode sceptique, que ce mode naturel d'ailleurs soit celui de la science ou bien de la vie quotidienne; les deux sont pour Quine en continuité. On opposera donc la nature à la philosophie, en conservant l'idée que cette nature n'est soutenue par rien d'autres qu'elle-même, ce qui est un des persuasions des pensées naturalistes ${ }^{6}$. Ainsi cet acquiescement au langage qui a suscité la méfiance des lecteurs de Quine, s'éclaire quant on la rapporte à la tradition sceptique d'opposition entre croyance et doute philosophique. On peut certes encore s'interroger sur la nature de l'assentiment humien aux croyances naturelles, mais

\footnotetext{
${ }^{6} \mathrm{Cf}$. le titre éclairant d'un des derniers articles de Quine, «Naturalism, or Living Within One's Means » [13].
} 
on peut en tout cas voir comment certaines étapes quiniennes délicates trouvent en réalité un écho dans la tradition, plus particulièrement dans la philosophie de la croyance de Hume.

\section{CONCLUSION}

Notre propos ici n'est pas de juger en général de la cohérence de philosophies empiristes cherchant à la fois à défaire une garantie, et à maintenir les concepts en jeu dans toute leur validité. Nous considérons simplement les proximités. Voici en particulier une formulation des problèmes de la philosophie de Hume proposée par R. Fogelin dans son essai Hume's Skepticism in the Treatise of Human Nature, qui caractérise sa démarche d'une manière convenant tout à fait à l'approche quinienne, de telle sorte qu'on introduisant ces tournants linguistique et holiste posé par l'imagerie historique de Quine, nous pouvons effectivement lire une partie de sa philosophie comme une reformulation amendée des problèmes de Hume. La succession des étapes de l'argumentation humienne obéit au modèle suivant selon Fogelin :

(i) un argument montrant qu'une croyance n'a pas de fondation rationnelle, (ii) une tentative d'éviter le scepticisme conceptuel de telle sorte que l'objet de croyance n'est pas perdu, et (iii) une explication naturaliste de l'origine de cette croyance. [2, pp. 78-79]

Fogelin oppose ce qu'il appelle scepticismes épistémologique et conceptuel ${ }^{7}$ d'une manière $^{\prime}$ éclairant également le travail de Quine :

Un scepticisme épistémologique accepte un système de croyances comme intelligible, mais remet en question les fondements qu'on suppose à ces croyances. Un scepticisme conceptuel remet en question l'intelligibilité même de ce système de croyance. [2, p. 6]

Fogelin, dans son premier chapitre, critique la tentation, qu'il trouve par exemple défendue par Ayer, de placer Hume à la tête de la tradition du positivisme logique. Et il défend cette position en montrant avant tout la distance entre les critères empiriques de signifiance (meaningfulness) de l'empirisme logique et les critères empiriques de clarté et précision qui sont ceux de Hume. La différence principale que souligne Fogelin est le caractère secondaire du souci linguistique chez Hume, alors qu'il est primordial dans l'empirisme logique. Nous avons vu en quoi ce n'est pas tellement cela qui importe dans l'établissement de la filiation. On peut partager les doutes de Fogelin, par contre, quand il souligne le refus d'arguments sceptiques conceptuels chez Hume, car nous retrouvons ce refus chez Quine, alors qu' une partie de l'empirisme logique avant Quine mettait ces arguments d'inintelligibilité au centre de leur dissolution du problème de la réalité extérieure de l'objet. En réalité, si Hume doit être rapproché des développements récents de l'empirisme, ce serait plutôt au niveau de la critique de l'empirisme logique par Quine. Citons, pour conclure, ce désaccord éclairant de Ayer avec Hume, et donc Quine :

Comment (...) parvenons-nous à une croyance garantie en l'existence d'objets physiques qui aient les propriétés perceptibles que nous leur reconnaissons normalement? Ma suggestion est que Hume lui-même fournit la meilleure réponse. Les phénomènes de constance et de cohérence, qu'il invoque pour expliquer comment nous tombons dans l'illusion de supposer que nos perceptions ont une existence continue et distincte, peuvent être considérés plutôt comme

\footnotetext{
${ }^{7}$ Fogelin pense par ailleurs que Hume ne parvient pas réellement à éviter le scepticisme conceptuel, et que cela conduit à des tensions irrésolues dans son œuvre.
} 
offrant un fondement adéquat pour une transformation imaginative des impressions des sens (...) en constituants du monde physique du sens commun. La seule erreur sérieuse que Hume ait faite était de supposer que les objets ainsi obtenus étaient des fictions. [1, p. 46]

La fiction humienne n'est bien entendu pas l'indétermination. Mais l'erreur de Hume, selon Ayer, est plutôt une anticipation, selon Quine, de ce que l'objet ne peut être considéré comme un ensemble cohérent et constant de perceptions empiriques données.

\section{RÉFÉRENCES}

[1] Alfred J. Ayer, Hume, Hill and Wang, 1980.

[2] Robert Fogelin, Hume's Skepticism in the Treatise of Human Nature, Routledge and Kegan Paul, 1985.

[3] David Hume, A Treatise of Human Nature, John Noon / Thomas Longman, 1739 / 1740. éd. citée : Penguin, Londres, 1985. Tr. fr. Traité de la nature humaine par P. Baranger et P. Saltel, Flammarion, Paris, 1995.

[4] S ANDRA LAUGIER, L'anthropologie logique de Quine, l'apprentissage de l'obvie, Vrin, 1992.

[5] Willard Van Orman Quine, From a Logical Point of View, Harvard University Press, 1953, $2^{\text {nde }}$ édition 1980. Tr. fr. D'un point de vue logique, S. Laugier (ed.), Vrin, Paris, 2003.

[6] Willard Van Orman Quine, «Identity, Ostention and Hypostasis», in From a Logical Point of View [5], pp. 65-79.

[7] Willard Van Orman Quine, Word and Object, MIT Press, 1960. Tr. fr. Le mot et la chose, J. Dopp et P. Gochet, Flammarion, Paris, 1978.

[8] Willard VAN ORMAN QUine, «Epistemology Naturalized», in Ontological Relativity and Other Essays [9].

[9] Willard Van Orman Quine, Ontological Relativity and Other Essays, Columbia University Press, 1969. Tr. fr. Relativité de l'ontologie et autres essais par J. Largeault, Aubier, Paris, 1977.

[10] Willard Van ORMan Quine, The Roots of Reference, Open Court, 1974.

[11] Willard Van Orman QUine, «Fives Milestones of Empiricism», in Theories and Things [12], pp. 67-72.

[12] Willard Van Orman Quine, Theories and Things, The Belknap Press, 1981.

[13] Willard Van Orman Quine, «Naturalism; or, Living Within One's Means», Dialectica 49 (1995), pp. 251-261. 